\title{
Band structure of a two-dimensional electron gas in the presence of two-dimensional electric and magnetic modulations and a perpendicular magnetic field
}

\author{
X. F. Wang, ${ }^{1}$ P. Vasilopoulos, ${ }^{1}$ and F. M. Peeters ${ }^{2}$ \\ ${ }^{1}$ Department of Physics, Concordia University, 1455 de Maisonneuve Boulevard. West, Montréal, Québec, Canada, H3G 1 M8 \\ ${ }^{2}$ Departement Natuurkunde, Universiteit Antwerpen (Campus Drie Eiken), Universiteitsplein 1, B-2610 Antwerpen, Belgium
}

(Received 2 February 2004; revised manuscript received 11 May 2004; published 15 October 2004)

\begin{abstract}
Two-dimensional (2D) periodic electric modulations of a 2D electron gas split each Landau level into the well-known butterfly-type spectrum described by a Harper-type equation multiplied by an envelope function. This equation is slightly modified for $2 \mathrm{D}$ magnetic modulations but the spectrum remains qualitatively the same. The same holds if both types of modulations are present. The modulation strengths do not affect the structure of the butterfly-type spectrum, they only change its scale or its envelope. The latter is described by the ratio $\alpha$ of the flux quantum $h / e$ to the flux per unit cell. Exact numerical and approximate analytical results are presented for the energy spectrum as a function of the magnetic field. For integer $\alpha$ the internal structure collapses into a band for all cases. The bandwidth at the Fermi energy depends on the modulation strength, the electron density, and, when both modulations are present, on the phase difference between them. In the latter case if the modulations have a $\pi / 2$ phase difference, the bandwidth at the Fermi energy is nearly independent of the magnetic field and the commensurability oscillations of the diffusive contribution to the resistivity disappear.
\end{abstract}

DOI: $10.1103 /$ PhysRevB.70.155312

PACS number(s): 73.20.At, 73.61.-r, 73.43.Qt

\section{INTRODUCTION}

In the last 15 years the magnetotransport of the twodimensional electron gas (2DEG), subjected to periodic potential modulations, has attracted considerable experimental and theoretical attention. For one-dimensional (1D) modulations oscillations of the magnetoresistivity tensor $\rho_{\mu \nu}$ have been observed, ${ }^{1}$ at low magnetic fields $B$, distinctly different in period and temperature dependence from the usual Shubnikov-de Haas ones observed at higher $B$. These oscillations reflect the commensurability between two length scales: $^{2}$ the cyclotron diameter at the Fermi level $2 R_{c}$ $=2 \sqrt{2 \pi n_{e}} \ell^{2}$, where $n_{e}$ is the electron density, $\ell$ the magnetic length, and $a$ the period of the potential modulation.

The situation is similar but less clearcut for 2D electric modulations from both a theoretical ${ }^{3,4}$ and an experimental ${ }^{5}$ point of view. In general, for 2D modulations a tight-binding treatment shows that each Landau level exhibits the wellknown butterfly-type spectrum described by a Harper-type equation. For sinusoidal modulations the energy spectrum resulting from the numerical solution of this equation shows, when the energy $E$ is measured in units of $V_{\mu} F_{n}\left(u_{\mu}\right), \mu=x, y$, with $V_{\mu}$ the modulation potential and $F_{n}\left(u_{\mu}\right)$ the Laguerre polynomial, a nontrivial structure as a function of $\alpha=\Phi / \Phi_{0}$, where $\Phi_{0}=h / e$ is the flux quantum and $\Phi$ the flux per unit cell: For $\alpha=p / q$ rational, with $q, p$ integers and relative prime, each Landau level is split into $p$ subbands. ${ }^{6}$ To our knowledge, despite various efforts ${ }^{7}$ there is no conclusive experimental evidence for this structure yet presumably because the small gaps between these subbands are closed due to disorder in samples of not exceptionally high mobilities. By neglecting those small gaps we recently accounted ${ }^{8}$ for the observed ${ }^{9}$ oscillations in the amplitude of the commensurability oscillations, as a function of the flux through the unit cell of the 2D modulation lattice, that could not be fully explained by earlier semiclassical theories. ${ }^{3}$ The high resistivity peaks observed for $\alpha$ integer were due to collisional contributions and show up only for short-period modulations.

In view of recent work on 1D magnetic modulations, ${ }^{10,11}$ it is of interest to present a tight-binding treatment of $2 \mathrm{D}$ magnetic modulations in order to see the similarity and the differences between the former and the latter as well as between 2D electric and magnetic modulations. So far we are aware only of the work of Ref. 12 which considered only a 2D magnetic modulation and did not show any spectra as a function of $\alpha=q / p$. In addition, we would like to present such a treatment when both types of modulations are present. The reason for considering the latter case is that from experimentally known methods of producing a magnetic modulation one expects that the magnetic ${ }^{10}$ or superconducting stripes,${ }^{11}$ periodically placed on top of a $2 \mathrm{DEG}$, also act like electrical gates and induce an electric modulation of the 2DEG. As shown in our previous work on transport ${ }^{13}$ and the related band structure, ${ }^{14}$ the phase difference between the two 1D modulations can have important consequences and even suppress the Weiss oscillations; this prediction was later confirmed experimentally. ${ }^{10}$ We show here that similar effects must occur for $2 \mathrm{D}$ modulations with a $\pi / 2$ phase difference since the bandwidth at the Fermi energy is nearly independent of the magnetic field and the commensurability oscillations of the diffusive contribution to the resistivity disappear. Further, as for 1D modulations ${ }^{13}$ the bandwidth at the Fermi energy for a purely electric modulation oscillates, as a function of the magnetic field, in antiphase with that for a purely magnetic one. In addition, we show how a small broadening can close the very small gaps in the density of states for $\alpha=q / p$ rational.

In the next section we derive the relevant Harper-type equations and solve them exactly, for $\alpha$ rational, and analyti- 
cally, for $\alpha$ integer, to obtain the one-electron eigenfunctions and eigenvalues. We also present the density of states and the bandwidth at the Fermi energy. Various numerical results are presented in Sec. III and concluding remarks in Sec. IV.

\section{FORMALISM}

We consider a 2DEG in the $(x, y)$ plane under a perpendicular magnetic field $B$, modulated weakly and periodically along the $x$ and $y$ directions, and accompanied by a 2D electric modulation of the same period. In Fourier space the total electric and magnetic fields can be expressed, respectively, by a vector potential

$$
\mathbf{A}(\mathbf{r})=\hat{\mathbf{x}} \sum_{\mathbf{g}} A_{\mathbf{g}}^{x} e^{i \mathbf{g} \cdot r}+\hat{\mathbf{y}}\left(B x+\sum_{\mathbf{g}} A_{\mathbf{g}}^{y} e^{i \mathbf{g} \cdot r}\right)
$$

and by a scalar potential

$$
\phi(\mathbf{r})=\sum_{\mathbf{g}} \phi_{\mathbf{g}} e^{i \mathbf{g} \cdot r} .
$$

Here $a_{x}=2 \pi / K_{x}$ and $a_{y}=2 \pi / K_{y}$ are the lattice periods of the imposed modulations and $\mathbf{g}=\hat{\mathbf{x}} n_{x} K_{x}+\hat{\mathbf{y}} n_{y} K_{y}$ is the corresponding reciprocal lattice vector with $n_{x}, n_{y}$ integers. For $\nabla \cdot \mathbf{A}=\mathbf{0}$, which can be satisfied in most practical situations, we have $A_{\mathbf{g}}^{x} g_{x}+A_{\mathbf{g}}^{y} g_{y}=0$ and the magnetic field reads

$$
\mathbf{B}=\nabla \times \mathbf{A}=\hat{\mathbf{z}}\left(B+\sum_{\mathbf{g}} B_{\mathbf{g}}^{z} e^{i \mathbf{g} \cdot r}\right),
$$

with $B_{\mathbf{g}}^{z}=i g_{x} A_{\mathbf{g}}^{y}-i g_{y} A_{\mathbf{g}}^{x}$ the Fourier component of the magnetic modulation.

Neglecting the spin of the electron and the Zeeman term, the one-electron Hamiltonian of the 2DEG reads

$$
\begin{aligned}
H= & {[\mathbf{p}+e \mathbf{A}(\mathbf{r})]^{2} / 2 m^{*}+V(\mathbf{r})=p_{x}^{2} / 2 m^{*}+\left(p_{y}+e B x\right)^{2} / 2 m^{*} } \\
& +\frac{e}{m^{*}} \sum_{\mathbf{g}} e^{i \mathbf{g} \cdot r}\left(A_{\mathbf{g}}^{x} p_{x}+A_{\mathbf{g}}^{y} p_{y}+e B x A_{\mathbf{g}}^{y}\right)+\frac{e^{2}}{2 m^{*}} \sum_{\mathbf{g}} D_{\mathbf{g}} e^{i \mathbf{g} \cdot r} \\
& +\sum_{\mathbf{g}} V_{\mathbf{g}} e^{i \mathbf{g} \cdot r},
\end{aligned}
$$

where $\mathbf{p}$ is the momentum operator, $D_{\mathbf{g}}=\sum_{\mathbf{g}^{\prime}}\left(A_{\mathbf{g}-\mathbf{g}^{\prime}}^{x} A_{\mathbf{g}^{\prime}}^{x}\right.$ $\left.+A_{\mathbf{g}-\mathbf{g}^{y}}^{y}, A_{\mathbf{g}^{\prime}}^{y}\right)$ is the square term of the vector potential, and $V_{\mathrm{g}}=-e \phi_{\mathrm{g}}$ is the Fourier transform of the electric potential.

In the absence of both modulations, i.e., for $B_{\mathbf{g}}^{z}=0$ and $\phi_{\mathrm{g}}=0$, the system has an energy spectrum of Landau levels $E_{n}=\hbar \omega_{c}(n+1 / 2)$. In the Landau gauge, the corresponding wave functions are

$$
\left\langle\mathbf{r} \mid n, k_{y}\right\rangle \equiv \psi_{n, k_{y}}=L_{y}^{-1 / 2} \exp \left(i k_{y} y\right) \phi_{n}\left(x+x_{0}\right)
$$

with $x_{0}=l^{2} k_{y}, l^{2}=\hbar / e B$, and $\phi_{n}(x)$ the normalized harmonic oscillator function. Constraining the center of the cyclotron orbit $-x_{0}$ to be within the sample and using periodic boundary condition along $y$, gives the degeneracy of the Landau levels as $L_{x} L_{y} / 2 \pi l^{2}=N$, with $L_{x}$ and $L_{y}$ the sample dimensions. We choose $N$ to be an integer.

\section{A. Tight-binding treatment}

In the presence of weak 1D modulation, when coupling between different Landau levels due to modulation is negli- gible, the Landau levels are broadened into bands and their bandwidth oscillates with the uniform field $B$. The eigenstates of the system and its energy spectrum can be calculated using perturbation theory ${ }^{13}$ or following a tight-binding treatment. ${ }^{15}$

For 2D modulations, the situation is much more complicated and in general the eigenstates cannot be the same as those without modulation. A tight-binding method ${ }^{16}$ used by Hofstadter ${ }^{6}$ to treat a $2 \mathrm{DEG}$ with strong electric modulation but weak perpendicular magnetic field results in a "butterfly"-type energy spectrum. Neglecting coupling between different Landau levels, we can express the eigenstates as linear combinations of the states without modulation for each Landau level. ${ }^{17}$ In the following, we will show that using the same method, the electron states in the presence of both electric and magnetic modulations can also be obtained. Notice, however, that in this method the parameter $\alpha$ is replaced by its inverse, ${ }^{18}$ i.e., in what follows we will have $\alpha$ $=\Phi_{0} / \Phi=q / p$.

For weak modulations, i.e., for $B_{\mathbf{g}}^{z} \ll B$ and $V_{g} \ll \hbar \omega_{c}$, we neglect the coupling between Landau levels. Then from Eq. (4) we obtain, for the $n$th Landau level, the matrix elements

$$
\left\langle n, k_{y}^{\prime}|H| n, k_{y}\right\rangle=E_{n} \delta_{k_{y}^{\prime}, k_{y}}+\sum_{\mathbf{g}} U_{\mathbf{g}} e^{-i l^{2} g_{x}\left(2 k_{y}+g_{y}\right) / 2} \delta_{k_{y}^{\prime}, k_{y}+g_{y}},
$$

where $U_{\mathbf{g}}$ is the effective potential

$$
U_{\mathbf{g}}=V_{\mathbf{g}} F_{n}(Q)+(e \hbar / m *) B_{\mathbf{g}}^{z} G_{n}(Q)+\left(e^{2} / 2 m *\right) D_{\mathbf{g}} F_{n}(Q),
$$

with $F_{n}(x)=\exp (-x / 2) L_{n}(x), G_{n}(x)=-(\partial / \partial x) F_{n}(x), Q=l^{2}\left(g_{x}^{2}\right.$ $\left.+g_{y}^{2}\right) / 2$, and $L_{n}(x)$ the Laguerre polynomial of order $n$.

From Eq. (6) we see that if only the modulation along the $x$ direction is present, i.e., for $g_{y}=0$, there results only a level broadening for each state but no coupling between different states. As noticed earlier, ${ }^{9,15}$ the modulation along the $y$ direction, however, introduces a coupling between the states $\left\langle n, k_{y}\right|$ and $\left\langle n, k_{y}+K_{y}\right|$. This leads to the folding of the energy band and the formation of a Brillouin zone along the $y$ direction. Then we can assume a wave function of the form: $\| n, \zeta\rangle=\Sigma_{\lambda} c_{\lambda}(n, \zeta)\left|n, k_{y}+\lambda K_{y}\right\rangle$ with $\lambda=1,2, \ldots, M$ for integer $M=N a_{y} / L_{y}$. The secular equation $\left.\left.H \| n, \zeta\right\rangle=\mathcal{E}_{n, \zeta} \| n, \zeta\right\rangle$ then becomes

$$
\sum_{\lambda}\left\{H_{\lambda^{\prime} \lambda}-\left(\mathcal{E}_{n, \zeta}-E_{n}\right) \delta_{\lambda^{\prime} \lambda}\right\} c_{\lambda}(n, \zeta)=0,
$$

with $\quad H_{\lambda^{\prime} \lambda}=\sum_{\mathbf{g}} \delta_{\lambda^{\prime}, \lambda+g_{y} / K_{y}} U_{\mathbf{g}} \exp \left[-i l^{2} g_{x}\left(2 k_{y}+\left(\lambda^{\prime}+\lambda\right) K_{y}\right) / 2\right]$. For sinusoidal modulations and rational values of $\alpha$ Eq. (8) becomes an extension of Harper's equation, see below.

For a rational value of

$$
\alpha=\Phi_{0} / \Phi=2 \pi l^{2} / a_{x} a_{y}=q / p
$$

with $q$ and $p$ integers and relative prime, the $M$-dimensional matrix $H_{\lambda, \lambda^{\prime}}$ satisfies the periodic condition $H_{\lambda+p, \lambda^{\prime}+p}=H_{\lambda, \lambda^{\prime}}$. Due to the peculiar character of the harmonic function centered at $x_{0}=l^{2} k_{y}$, this corresponds to an effective spatial periodicity of the Hamilitonian with period $p l^{2} K_{y}=q a_{x}$ and Bloch-type extensive states along the $x$ direction emerge as a 
general property of the superlattice. It is convenient ${ }^{17}$ to introduce a Bloch-type basis $\|\left|n, s, k_{x}, k_{y}\right\rangle$ with a quantum number $k_{x}$, the momentum of electrons along the $x$ direction,

$$
\left.\| n, s, k_{x}, k_{y}\right\rangle=\left(N_{x}\right)^{1 / 2} \sum_{\beta=1}^{N_{x}} e^{-i l^{2} k_{x}\left[k_{y}+(\beta p+s) K_{y}\right]}\left|n, k_{y}+(\beta p+s) K_{y}\right\rangle,
$$

where $N_{x}=L_{x} / q a_{x}$ is assumed an integer by choosing $L_{x}$.

In this basis the eigenstate of the Hamiltonian reads

$$
\left.\left.\left.\| n, j, k_{x}, k_{y}\right\rangle\right\rangle=\sum_{s=1}^{p} u_{s}\left(n, j, k_{x}, k_{y}\right) \| n, s, k_{x}, k_{y}\right\rangle .
$$

Since $u_{s}(\ldots)$ obeys the relation $u_{s+p}\left(n, j, k_{x}, k_{y}\right)$ $=u_{s}\left(n, j, k_{x}, k_{y}\right)$, it is a periodic function of $s$ and the $M$ $\times M$ Hamiltonian matrix is reduced into a $p \times p$ one with elements

$$
\begin{aligned}
{\left[h_{n}^{(p)}(k)\right]_{s^{\prime}, s}=} & \sum_{\mathbf{g}} \tilde{\delta}_{\left[g_{y} / K_{y}\right], s^{\prime}-s}^{(p)} U_{n, \mathbf{g}} \exp \left[-i l^{2}\left(g_{x} k_{y}-g_{y} k_{x}+s g_{x} K_{y}\right.\right. \\
& \left.\left.+g_{x} g_{y} / 2\right)\right],
\end{aligned}
$$

and

$$
\widetilde{\delta}_{\left[g_{y} / K_{y}\right], s^{\prime}-s}^{(p)}= \begin{cases}1 & \text { if }\left[g_{y} / K_{y}\right]=s^{\prime}-s \text { or } s^{\prime}-s+p \\ 0 & \text { otherwise }\end{cases}
$$

$\left[g_{y} / K_{y}\right] \quad$ is $\quad g_{y} / K_{y} \quad$ modulo $p, \quad k_{x} \in\left[0,2 \pi / q a_{x}\right], \quad k_{y}$ $\in\left[0,2 \pi / a_{y}\right]$, and $s=1,2, \ldots, p$. Each Landau level is split into $p$ minibands with energy denoted by $\mathcal{E}_{n, s}^{(p)}\left(k_{x}, k_{y}\right)$.

In this paper we will discuss in detail the energy spectrum and the density of states (DOS) of a 2DEG in a constant magnetic field $B$ with a weak magnetic modulation $\mathbf{B}$ $=\left[e m * \omega_{x} \cos \left(K_{x} x\right)+e m * \omega_{y} \cos \left(K_{y} y\right)\right] \hat{\mathbf{z}}$ and a weak electric modulation of the potential $V=V_{x} \cos \left(K_{x} x+\varphi_{x}\right)+V_{y} \cos \left(K_{y} y\right.$ $\left.+\varphi_{y}\right)$ with $\varphi_{x}, \varphi_{y}$ the phase differences between the respective components of the potential. The corresponding effective potential is found to be

$$
\begin{aligned}
U_{n}= & \sum_{\mathbf{g}} U_{n, \mathbf{g}} e^{i \mathbf{g} \cdot r}=V_{x} F_{n}\left(u_{x}\right) \\
& \times \cos \left(K_{x} x+\varphi_{x}\right)+V_{y} F_{n}\left(u_{y}\right) \cos \left(K_{y} y+\varphi_{y}\right) \\
& +\hbar \omega_{x} G_{n}\left(u_{x}\right) \cos \left(K_{x} x\right)+\hbar \omega_{y} G_{n}\left(u_{y}\right) \cos \left(K_{y} x\right) \\
& +\epsilon_{x}\left[1-F_{n}\left(4 u_{x}\right) \cos \left(2 K_{x} x\right)\right]+\epsilon_{y}\left[1-F_{n}\left(4 u_{y}\right) \cos \left(2 K_{y} y\right)\right],
\end{aligned}
$$

with $u_{\mu}=l^{2} K_{\mu}^{2} / 2$ and $\epsilon_{\mu}=m * \omega_{\mu}^{2} / 4 K_{\mu}^{2}, \quad \mu=x, \quad y$. With $\mathcal{E}_{n, s}^{(p)}\left(k_{x}, k_{y}\right) \equiv \mathcal{E}_{n, \zeta}$ the corresponding secular equation Eq. (8) becomes

$$
\begin{aligned}
& -\frac{\epsilon_{y}}{2} F_{n}\left(4 u_{y}\right)\left[C_{\lambda-2}+C_{\lambda+2}\right]+\left\{\epsilon_{x}+\epsilon_{y}-\epsilon_{x} F_{n}\left(4 u_{x}\right) \cos \left[2 l ^ { 2 } K _ { x } \left(k_{y}\right.\right.\right. \\
& \left.\left.\quad+\lambda K_{y}\right)\right]+\hbar \omega_{x} G_{n}\left(u_{x}\right) \cos \left[l^{2} K_{x}\left(k_{y}+\lambda K_{y}\right)\right] \\
& \left.\quad+V_{x} F_{n}\left(u_{x}\right) \cos \left[l^{2} K_{x}\left(k_{y}+\lambda K_{y}\right)-\varphi_{x}\right]-\left(\mathcal{E}_{n, \zeta}-E_{n}\right)\right\} C_{\lambda}
\end{aligned}
$$

$$
\begin{aligned}
& +\frac{1}{2}\left[\hbar \omega_{y} G_{n}\left(u_{y}\right)+V_{y} F_{n}\left(u_{y}\right) e^{i \varphi_{y}}\right] C_{\lambda-1}+\frac{1}{2}\left[\hbar \omega_{y} G_{n}\left(u_{y}\right)\right. \\
& \left.+V_{y} F_{n}\left(u_{y}\right) e^{-i \varphi_{y}}\right] C_{\lambda+1}=0 .
\end{aligned}
$$

For $\alpha=2 l^{2} K_{x} K_{y}=q / p$ rational, with $p, q$ integers and relative prime, the exact solution of this difference equation is obtained numerically by rewriting it in the form of a matrix, ${ }^{6,17}$ of dimension $p$, and solving the resulting eigenvalue problem with the details given above. It will be shown below.

For a pure electric modulation Eq. (15) holds with the terms $\propto \epsilon_{\mu}$ and $\propto \omega_{\mu}$ set equal to zero while for a pure magnetic modulation the terms $\propto V_{\mu}$ vanish; in either case we set $\phi_{\mu}=0$. Comparing these two cases, we see that Eq. (15) should lead to the same overall spectrum if $\hbar \omega_{\mu} G_{n}\left(u_{\mu}\right)$ $=V_{\mu} F_{n}\left(u_{\mu}\right)$ provided we neglect the very small quadratic terms $\propto \boldsymbol{\epsilon}_{\mu}$. Examples of these spectra will be shown in Sec. III.

\section{B. Results for $\alpha$ integer}

For $\alpha=2 l^{2} K_{x} K_{y}$ integer the dependence of the quantity $\{\ldots\}$ in Eq. (15) on $\lambda$ drops out since $\lambda$ is an integer. Then Eq. (15) admits the exponential solutions $C_{\lambda}=C_{0} \exp (i \xi \lambda)$; the resulting energy eigenvalue is

$$
\begin{aligned}
\mathcal{E}_{n}^{(1)}\left(k_{x}, k_{y}\right)= & E_{n}+\epsilon_{x}\left[1-F_{n}\left(4 u_{x}\right) \cos 2 \eta\right] \\
& +\epsilon_{y}\left[1-F_{n}\left(4 u_{y}\right) \cos 2 \xi\right]+\hbar \omega_{x} G_{n}\left(u_{x}\right) \cos \eta \\
& +\hbar \omega_{y} G_{n}\left(u_{y}\right) \cos \xi+V_{x} F_{n}\left(u_{x}\right) \cos \left(\eta-\varphi_{x}\right) \\
& +V_{y} F_{n}\left(u_{y}\right) \cos \left(\xi+\varphi_{y}\right),
\end{aligned}
$$

where $\xi=k_{x} \ell^{2} K_{y}$ and $\eta=k_{y} \ell^{2} K_{x}$. This is a simple sinusoidal band as when only one modulation is present. ${ }^{8}$ The corresponding eigenvectors are labeled with the additional quantum number $\xi(0 \leqslant \xi \leqslant 2 \pi):\left|\psi_{n k_{y} \xi}\right\rangle=C_{0} \Sigma_{p} \exp (i \xi \lambda) \mid n, k_{y}$ $\left.+\lambda K_{y}\right\rangle$. The orthonormality condition gives $\xi=k_{x} \ell^{2} K_{y}$ and $C_{0}=\ell\left(K_{y} / L_{x}\right)^{1 / 2}$ by normalization.

If we set $V_{x}=V_{y}=0$ in Eq. (16), we see that the only difference from the case of a pure electric 2D modulation is the presence of the terms $\epsilon_{\mu}$, which are proportional to $\omega_{\mu}^{2}$, on the first line of Eq. (16); however, these terms are very weak compared to those on the second and third line $\propto \omega_{\mu}$. Further, it is instructive to contrast the spectrum, given by Eq. (16), for a pure electric 2D modulation $\left(\hbar \omega_{\mu}=0\right)$ with that for a pure magnetic 2D modulation $\left(V_{\mu}=0\right)$ as done for Eq. (15). We then see explicitly that the two spectra are the same provided $\hbar \omega_{\mu} G_{n}\left(u_{\mu}\right)=V_{\mu} F_{n}\left(u_{\mu}\right)$. This means that the results of a pure electric modulation can be mimicked by those of a pure magnetic one and vice versa. Notice, however, that this equivalence does not hold for those values of $u_{\mu}$ for which the factors $G_{n}\left(u_{\mu}\right)$ or $F_{n}\left(u_{\mu}\right)$ vanish since they are not the same in both cases. The same conclusion was reached in Ref. 12 with the corresponding spectrum evaluated by first-order perturbation theory.

In the absence of modulations we have the discrete Landau levels $E_{n}$. In their presence Eq. (16) shows that these levels are broadened into the bands $\mathcal{E}_{n}^{(1)}\left(k_{x}, k_{y}\right)$ with explicit sinusoidal dependence on $k_{x}$ and $k_{y}$. If we neglect the very 
small terms $\propto \epsilon_{\mu}$ on the first line and take $\varphi_{x}=\varphi_{y}=0$ for simplicity, their bandwidth is given by $\Delta E_{n}\left(u_{x}, u_{y}\right)=2\left[V_{x} F_{n}\left(u_{x}\right)\right.$ $\left.+V_{y} F_{n}\left(u_{y}\right)+\hbar \omega_{x} G_{n}\left(u_{x}\right)+\hbar \omega_{y} G_{n}\left(u_{y}\right)\right]$ and it oscillates with the field $B$. The related velocity components $\left(v_{\mu}=(1)\right.$ $\left.\hbar)\left(\partial \mathcal{E}_{n}^{(1)}\left(k_{y}, k_{x}\right) / \partial k_{\mu}\right), \mu=x, y\right)$ resulting from Eq. (16) are

$$
\begin{aligned}
v_{x}= & -\left(l^{2} K_{y} / \hbar\right)\left[V_{y} F_{n}\left(u_{y}\right) \sin \left(\xi+\varphi_{y}\right)+\hbar \omega_{y} G_{n}\left(u_{y}\right) \sin \xi\right. \\
& \left.-2 \epsilon_{y} F_{n}\left(4 u_{y}\right) \sin 2 \xi\right], \\
v_{y}= & -\left(l^{2} K_{x} / \hbar\right)\left[V_{x} F_{n}\left(u_{x}\right) \sin \left(\eta-\varphi_{x}\right)+\hbar \omega_{x} G_{n}\left(u_{x}\right) \sin \eta\right. \\
& \left.-2 \epsilon_{x} F_{n}\left(4 u_{x}\right) \sin 2 \eta\right] .
\end{aligned}
$$

The broadening of the Landau levels into bands that gives rise to these velocity components has important consequences for transport ${ }^{4}$ especially when the fine structure of the exact energy spectrum is not resolved due to disorder. ${ }^{8}$ The main consequence is a nonvanishing diffusive contribution to the conductivity which is absent when the modulations are not present.

In the following we will show that we can use the expression of the energy spectrum Eq. (16) and the velocity operators Eqs. (17) and (18) for $\alpha$ integer to approximately estimate the physical properties of the 2DEG system for any value of $\alpha$.

\section{Density of states}

The energy spectrum given by $\mathcal{E}_{n, s}^{(p)}\left(k_{x}, k_{y}\right)$ is qualitatively different from the unmodulated spectrum, given by $E_{n}$, and from the corresponding 1D modulation spectrum given by $E_{n}+F_{n}\left(u_{x}\right) \cos K_{x} x_{0}$. These differences are also reflected in the density of states (DOS) defined by

$$
D(E)=2 \sum_{n, s, k_{x}, k_{y}} \delta\left[E-\mathcal{E}_{n, s}^{(p)}\left(k_{x}, k_{y}\right)\right] .
$$

For a Lorenzian broadening of width $\Gamma$, the $\delta$ function in the above equation should be replaced by $(1 / \pi) \Gamma / \Gamma^{2}+[E$ $\left.-\mathcal{E}_{n, s}^{(p)}\left(k_{x}, k_{y}\right)\right]^{2}$.

The result for $\alpha$ integer is obtained by replacing $\mathcal{E}_{n, s}^{(p)}\left(k_{x}, k_{y}\right)$ by $\mathcal{E}_{n, s}^{(1)}\left(k_{x}, k_{y}\right)$ in Eq. (19). For a $2 \mathrm{D}$ modulation with rectangular symmetry, corresponding to Eq. (16), the DOS becomes

$$
\begin{aligned}
D(E)= & D_{0} \sum_{n=0}^{\infty} \int_{0}^{2 \pi} d \xi\left\{\left[V_{x} F_{n}\left(u_{x}\right)+\hbar \omega_{x} G_{n}\left(u_{x}\right)\right]^{2}-\left[E-E_{n}\right.\right. \\
& \left.\left.-\left\{V_{y} F_{n}\left(u_{y}\right)-\hbar \omega_{y} G_{n}\left(u_{y}\right)\right\} \cos \xi\right]^{2}\right\}^{-1 / 2}
\end{aligned}
$$

where $D_{0}=L_{y} L_{x} / \pi^{3} \ell^{2}$. The radicant in Eq. (20) must be positive.

\section{RESULTS AND DISCUSSION}

\section{A. In-phase, antiphase modulations}

The results presented so far are valid for rectangular modulations. However, as the various expressions and results become simpler for square modulations, we will consider only the latter. For a square modulation where $K_{x}=K_{y}=K$, $\varphi_{x}=\varphi_{y}=\varphi, V_{x}=V_{y}=V, \omega_{x}=\omega_{y}=\omega$, the secular Eq. (8), without the square terms $\propto \epsilon_{\mu}$ due to the magnetic modulation, is Harper's equation with $\varphi=0$ if the modulations are in phase and $\varphi=\pi$ if they are in antiphase; in these cases Eq. (8) takes the form

$$
C_{\lambda-1} / 2+C_{\lambda+1} / 2+C_{\lambda} \cos \left(\lambda l^{2} K^{2}+K x_{0}\right)-C_{\lambda} \epsilon_{n, \zeta}=0,
$$

where $\epsilon_{n, \zeta}=\left(\mathcal{E}_{n, \zeta}-E_{n}\right) /\left[V F_{n}(u) \pm \hbar \omega G_{n}(u)\right]$. The resulting energy spectrum is the "butterfly" spectrum $\epsilon_{n, s}^{(p)}\left(k_{x}, k_{y}\right)$ $\in[-2,2]$ modulated by an envelope function determined by the modulations

$$
\mathcal{E}_{n, s}^{(p)}\left(k_{x}, k_{y}\right)=E_{n}+\left[V F_{n}(u) \pm \hbar \omega G_{n}(u)\right] \epsilon_{n, s}^{(p)}\left(k_{x}, k_{y}\right),
$$

where the upper (lower) sign is for in-phase (antiphase) modulations.

The spectrum for $\alpha$ integer and the corresponding wave function were used previously ${ }^{8}$ as an approximation, for all values of the magnetic field, and successfully described magnetotransport of a $2 \mathrm{DEG}$ in the presence of a $2 \mathrm{D}$ electric modulation in the case that the fine structure of the exact spectrum is not resolved. This approximation implies the replacement of $\epsilon_{n, s}^{(p)}\left(k_{x}, k_{y}\right)$ by $\epsilon_{n, 1}^{(1)}\left(k_{x}, k_{y}\right)=\cos \xi+\cos \eta$ in Eq. (22), which becomes

$$
\mathcal{E}_{n, s}^{(p)}\left(k_{x}, k_{y}\right) \approx E_{n}+\left[V F_{n}(u) \pm \hbar \omega G_{n}(u)\right](\cos \xi+\cos \eta) .
$$

In the following the resulting spectrum will be referred to as approximate band structure or approximate spectrum.

The edge of the approximate band is the envelope of the exact energy spectrum. Employing the asymptotic expressions of the Laguerre polynomials for $n \gg 1$, the corresponding bandwidth is estimated as

$$
\Delta E_{n}(u) \simeq 4\left(\pi^{2} n u\right)^{-1 / 4} \tilde{V}\left(1+\widetilde{\delta}^{2}\right)^{1 / 2}|\sin (2 \sqrt{n u}-\pi / 4+\beta)|,
$$

with $\quad \tilde{\delta}=(\hbar \omega / \widetilde{V}) \sqrt{n / u}, \quad \tilde{V}=V \pm \hbar \omega / 4 u \quad$ and $\quad \beta$ $=\arctan [ \pm(\tilde{V} / \hbar \omega) \sqrt{u / n}]$, so that the flat band condition is $\beta$ $\approx m \pi+\pi / 4-2 \sqrt{n u}$ where $n$ is the Landau index and $u$ $=K^{2} l^{2} / 2$. For $1 / 4 u \ll \sqrt{n / u}$ or $\alpha \gg 16 n \pi$, which is valid for typical parameters discussed in this paper, we can further approximate $\tilde{V}$ with $V$ and $\tilde{\delta}$ with $\delta=(\hbar \omega / V) \sqrt{n / u}$. Because $\beta=\pi$ for a pure electric modulation and $\beta=0$ for a pure magnetic modulation, the two modulations are phase shifted and their interplay will influence the results of transport experiments.

In Fig. 1 we plot the envelope function of the energy spectrum for Landau levels $n=0$ to $n=10$ as a function of the cyclotron energy $\hbar \omega_{c}$. For the same modulation strength $V$ $=\hbar \omega_{0}=0.1 \mathrm{meV}$ the ratio between the band broadenings is determined by the value of $\delta$, cf. Eq. (24). For a period $a$ $=200 \mathrm{~nm}$, cf. Figs. 1(a) and 1(b), the curve $\delta=1$ is located near $E \approx 0.8 \mathrm{meV}$ and the broadening due to magnetic modulation is generally larger than that due to the electric one. Decreasing the period $a$, the $\delta=1$ curve is shifted to a higher energy, higher than $3 \mathrm{meV}$ for $a=80 \mathrm{~nm}$. This is the reason 


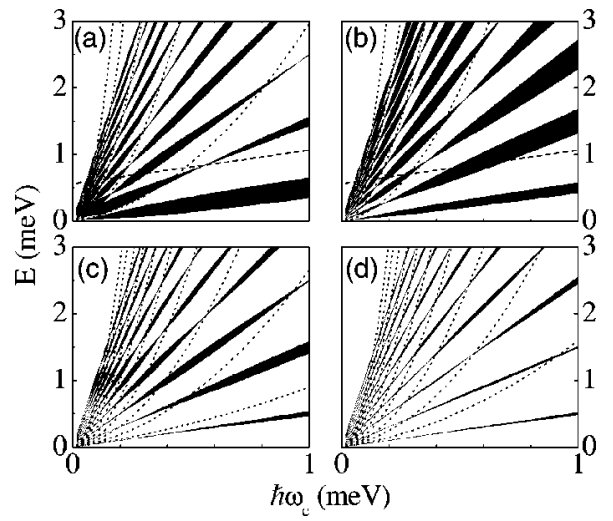

FIG. 1. Approximate band structure, for $n=0, \ldots, 10$, as a function of $\hbar \omega_{c}$ for square electric [(a) and (c)] or magnetic [(b) and (d)] modulations of long period $(a=200 \mathrm{~nm})$, in (a) and (b), and short period $(a=80 \mathrm{~nm})$ in (c) and (d). The crossings of the dotted curves with the Landau levels give the asymptotic flat-band positions and the dashed line, in panels (a) and (b), is the $\delta=1$ curve.

why the magnetic band broadening is narrower than the electric one for energies lower than $3 \mathrm{meV}$ as shown in Figs. 1(c) and $1(\mathrm{~d})$.

Figure 2 illustrates the envelope function as in Fig. 1 with both modulations having the same strength $V=\hbar \omega_{0}$ $=0.1 \mathrm{meV}$ and being in phase. In such a case, the modulation of longer period results in a broader bandwidth. This is opposite to that obtained from the band broadening in a common superlattice and results from the presence of the strong perpendicular magnetic field. In a common superlattice the miniband appears as a result of electron tunneling between adjacent quantum wells. The miniband width decreases when the superlattice period increases since the tunnel coupling weakens if the other parameters remain the same. However, here the level broadening stems mainly from the perturbative correction to the Landau energy of each state by the modulation. In Fig. 3 we show the resulting bandwidth of the tenth Landau level as a function of the modulation period for fixed magnetic field $B=0.5 \mathrm{~T}$ and the strength parameters shown in the caption. We see that on the average the width increases when the period increases, say by a factor of $s$ provided $s$ is such that $s a$ is not close to the point where the bandwidth vanishes. On the right panels of Fig. 3 we show the constant ( $\xi$ - or $\eta$-indepedent) term of the square of the velocity, given by Eqs. (17) and (18), versus the modulation period $a$ for the same magnetic field. If we neglect the very small terms $\propto \epsilon_{\mu}$,
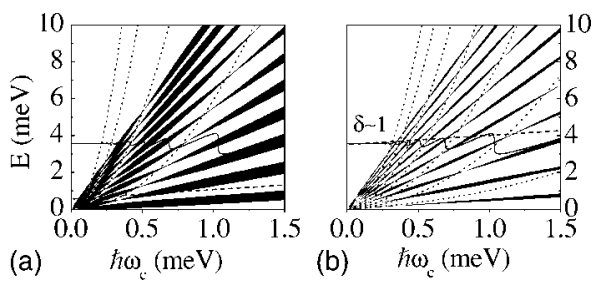

FIG. 2. The same as in Fig. 1 for both electric and magnetic in-phase modulations of long (a) and short (b) period. The thin solid curves show the Fermi energy for temperature $T=1 \mathrm{~K}$ and electron density $n_{e}=10^{11} \mathrm{~cm}^{-2}$.
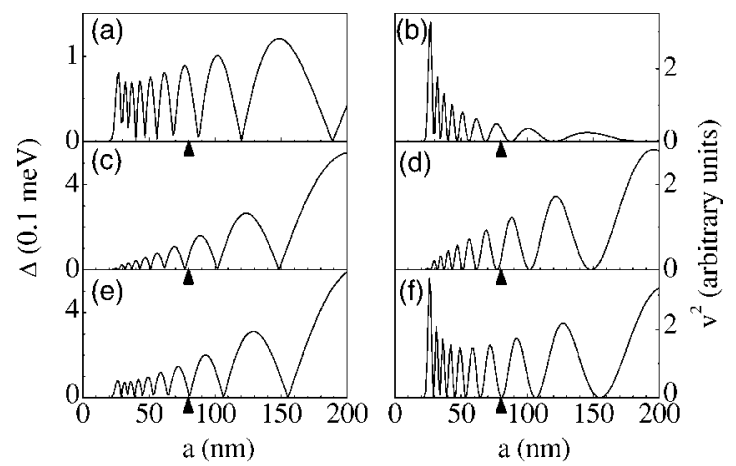

FIG. 3. Left panels: The approximate bandwidth $\Delta$ of the 10th Landau level as a function of the modulation period $a$ at magnetic field $B=0.5 \mathrm{~T}$ for modulation strengths (a) $V=0.1 \mathrm{meV}$ and $\hbar \omega$ $=0$, (b) $V=0$ and $\hbar \omega=0.1 \mathrm{meV}$, and (c) $V=\hbar \omega=0.1 \mathrm{meV}$. Right panels: The constant term of the square of the velocity in arbitrary units, given by Eqs. (17) or (18), versus the modulation period $a$ at magnetic field $B=0.5$. Panels (d), (e), and (g) correspond to panels (a), (b), and (c), respectively. The two periods used in Figs. 1 and 2 are $80 \mathrm{~nm}$, indicated by the triangle, and $200 \mathrm{~nm}$.

this term is equal to $\left[\left(\ell^{2} K_{y} / \hbar\right) V_{y} F_{n}\left(u_{y}\right)+\hbar \omega_{y} K_{y} G_{n}\left(u_{y}\right)\right]^{2} / 2$. This term gives by far the dominant contribution to the diffusive conductivity, given by the standard expression $\sigma_{\mu \nu}^{d i f}(0)=\left(\beta e^{2} / \Omega\right) \sum_{\zeta} f_{\zeta}\left(1-f_{\zeta}\right) \tau\left(E_{\zeta}\right) v_{\mu}^{\zeta} v_{\nu}^{\zeta}$, where $f$ is the FermiDirac function, $v_{\mu}^{\zeta}$ the velocity, and $\tau$ the relaxation time ${ }^{8}$ pertinent to a state $\zeta \equiv\left\{k_{x}, k_{y}, n\right\}$. With the integrals over $k_{x}$ and $k_{y}$ giving, for this term, just a constant prefactor near the Fermi level, we see that this contribution is mainly determined by the bandwidth. By contrasting the left and right panels one can see that this on-the-average increase of the bandwidth with the period is mainly due to the behavior of the contribution of the magnetic modulation to it. This behavior of the bandwidth and the antiphase between panels (a) and (b) on the left, and panels (d) and (c) on the right, are directly related to those of the Laguerre polynomials and their derivatives that appear in the factors $F_{n}$ and $G_{n}$.

If both modulations are present, their relative contribution to the energy broadening is determined by the value of $\delta$. The magnetotransport behavior of a $2 \mathrm{DEG}$ is directly related to the level broadening at the Fermi energy. Because the electric modulation dominates the low-energy broadeningand the magnetic modulation dominates the high-energy one, the transition from one energy range to another can be observed if we shift the Fermi energy through these ranges by changing the electron density. In Fig. 4 we plot the bandwidth at the Fermi energy for a period $a=80 \mathrm{~nm}$ as a function of the perpendicular magnetic field $B$. The bandwidth oscillations, resulting when only the electric modulation is present, are in antiphase with those resulting when only the magnetic one is present. This should show up in the commensurability oscillations of the magnetoresistance as it does for 1D modulations. ${ }^{13}$ If both modulations have the same strength, the bandwidth oscillations (solid curves) are similar to those due to the electric one at the lower electron density [Fig. 4(a)] and similar to those due to the magnetic one at the higher electron density [Fig. 4(d)]. The nonsmooth behavior of the bandwidth, when the field $B$ is varied, reflects that of 
(a)
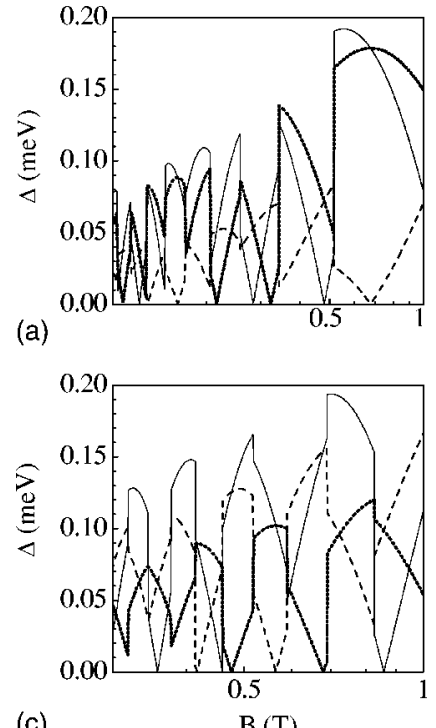

(c)

FIG. 4. The approximate bandwidth of the Landau level closest to the Fermi level as a function of the magnetic field for different electron densities $n_{e}=0.5$ (a), 1.0 (b), 2.0 (c), and $5.0 \times 10^{11} \mathrm{~cm}^{-2}$ (d). The solid curves denote broadening with both modulations present and of the same strength. The dotted (dashed) curves show the broadening when only the electric (magnetic) modulation is present.

the Fermi level, as shown, for instance, in Fig. 2. Upon changing the electron density the Fermi level crosses different Landau levels in a nonsmooth manner, cf. Fig. 2. For higher densities and/or longer periods more Landau levels are occupied and the oscillations are much smoother.

In Fig. 5 we plot the exact energy spectrum of the second Landau level, in units of $\hbar \omega_{c}$, as a function of $\hbar \omega_{c}$ for (a) only an electric modulation, (b) only a magnetic modulation, and (c) both electric and magnetic modulations all of period $a=80 \mathrm{~nm}$ and strength $V=\hbar \omega=0.1 \mathrm{meV}$. The positions of

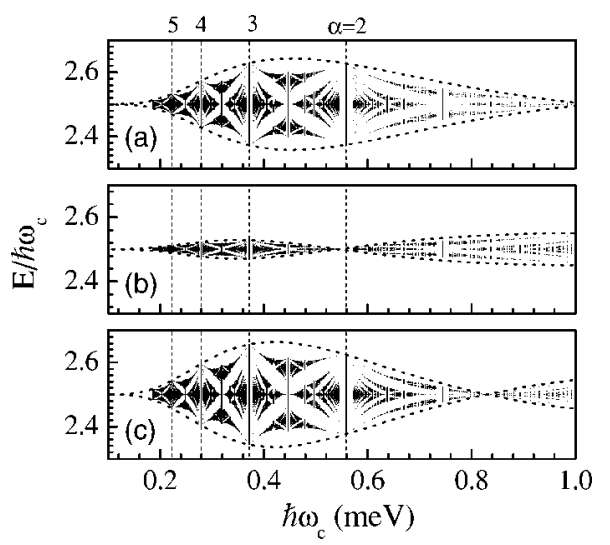

FIG. 5. Exact and approximate ( $\alpha$ integer) spectrum, in units of $\hbar \omega_{c}$, versus the cyclotron energy $\hbar \omega_{c}$ for the $n=2$ Landau level and a period $a=80 \mathrm{~nm}$. The upper panel is for an electric modulation, the middle one for a magnetic one, and the bottom one for both modulations present. Notice the continuous bands for $\alpha$ integer: In this case the exact, numerically obtained bandwidth coincides with the one obtained analytically.
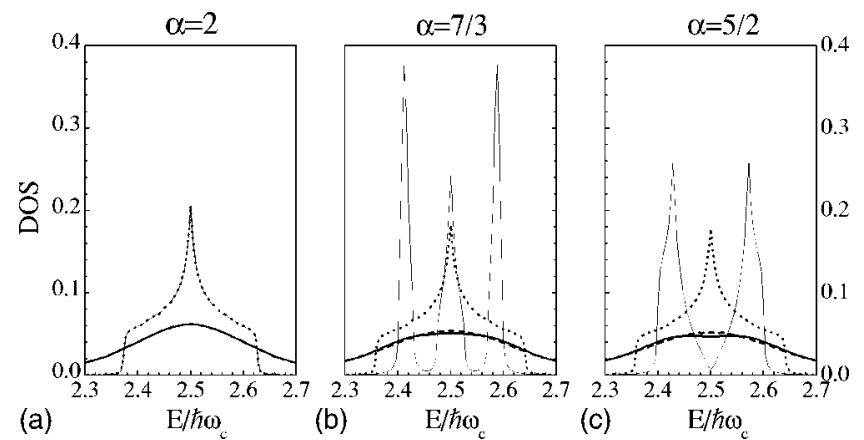

FIG. 6. Broadened DOS for the exact (thin solid curves: $\Gamma$ $=0.01 \mathrm{~K}$, thick curves: $\Gamma=0.5 \mathrm{~K}$ ) and the $\alpha$ integer spectrum approximation (dotted curves: $\Gamma=0.01 \mathrm{~K}$, dashed curves: $\Gamma=0.5 \mathrm{~K}$ ). The magnetic field is such that $\alpha=2$ in. (a), $\alpha=7 / 3$ in. (b), and $\alpha$ $=5 / 2$ in. (c). $V=\hbar \omega_{0}=0.1 \mathrm{meV}$.

$\alpha=q / p=2,3,4,5$ are marked by the dashed vertical lines. The exact spectrum is the same as Hofstadter's spectrum modulated by the envelope function (dotted curves in Fig. 5). For a rational value of $\alpha=q / p$ the spectrum is composed of $p$ minibands which touch each other for $\alpha$ half integer or $p$ $=2$. For $\alpha$ integer the spectrum is the sinusoidal band given by Eq. (23). The envelope function, given by the dotted curves, is the band edge of the $\alpha$ integer spectrum [Eq. (23)] used here for all values of $\alpha$. A negligible shift and the modification of the spectrum, due to the terms $\propto \epsilon_{\mu}$, are not shown in the figures.

In Fig. 6 we show the exact and approximate DOS for in-phase modulations and various values of $\alpha$. The other parameters are specified in the caption. The results coincide, as they should, for $\alpha=2$. Notice the horizontal scale and how a small level broadening $\Gamma$ closes the gaps of the exact DOS in panels (b)-(d).

\section{B. Out-of-phase modulations}

For arbitrary phase difference between the two modulations, the secular equation cannot be written simply as Harper's equation but as a more general one, cf. Eq. (15). However, the energy spectrum for $\alpha$ integer is still the envelope of the exact spectrum and the asymptotic bandwidth is

$$
\begin{aligned}
\Delta E_{n}(u)= & 4 V\left(\pi^{2} n u\right)^{-1 / 4}\left[1+\left(\delta^{2}-1\right) \sin ^{2}(2 \sqrt{n u}-\pi / 4)\right. \\
& -\delta \cos \phi \cos (4 \sqrt{n u})]^{1 / 2}
\end{aligned}
$$

where $\delta=(\hbar \omega / V)(n / u)^{1 / 2}$. At the Fermi energy we have $\delta\left(E_{f}\right)=a k_{F} \hbar \omega /(2 \pi V)$. If $\delta=1$ and $\varphi=\pi / 2$, we get a nearly constant bandwidth $4 V\left(\pi^{2} n u\right)^{-1 / 4}$.

In Fig. 7 we plot the energy of the $n=0, \ldots, 10$ Landau levels as a function of the magnetic field for equal-strength $(0.1 \mathrm{meV})$ modulations, of period $80 \mathrm{~nm}$, that are out of phase by $\pi / 2$. The dotted curve, i.e., $\delta=1$, shows a constant bandwidth that, as explained above, leads to a washout of the commensurability oscillations. The thin solid curve shows the Fermi level at $T=5 \mathrm{~K}$.

The amplitude of the bandwidth oscillations varies with the phase difference between the two modulations and/or with their relative strength. In Fig. 8 we plot the bandwidth 


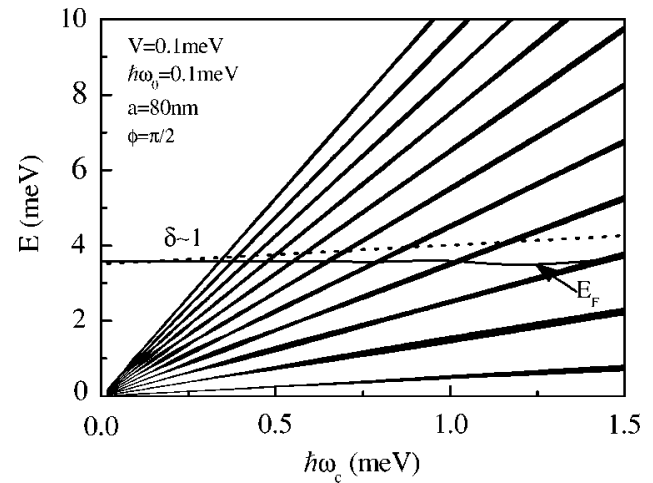

FIG. 7. Band structure with both modulations present and phase shifted by $\phi=\pi / 2$. The Fermi level at temperature $T=5 \mathrm{~K}$ is also indicated by the thin solid curve. The $\delta \sim 1$ curve shows a constant bandwidth leading to a washout of the commensurability oscillations of the diffusive contributions to the resistivity.

for equal modulation strengths, $V=\hbar \omega=0.1 \mathrm{meV}$, and different phases. As Fig. 8(b) shows, the amplitude can be greatly decreased when $\phi=\pi / 2$ and this implies a washout of the commensurability oscillations. Different from the in-phase or antiphase case (Fig. 4), we notice that the bandwidth increases more rapidly with magnetic field and that it does not reach zero at specific values of $B$. In Fig. 9 we show again the bandwidth for modulations phase shifted by $\pi / 2$ but with different relative strengths apart from panel (c), in which the two strengths are equal, and the amplitude of the oscillations minimal.

Finally, in Fig. 10 we plot the exact and approximate spectra of the $n=2$ Landau level, as a function of the magnetic field, for various phase differences between the two modulations as indicated. The approximate result, for $\alpha$ integer, is shown by dotted curves and is again the envelope function of the exact spectrum as for in-phase modulations.

\section{CONCLUSIONS}

We studied in detail the band structure of a 2DEG in the presence of weak 2D electric and magnetic modulations as function of an applied perpendicular magnetic field $B$. The tight-binding description shows a Hofstadter-type spectrum with an envelope function that is determined by the strengths
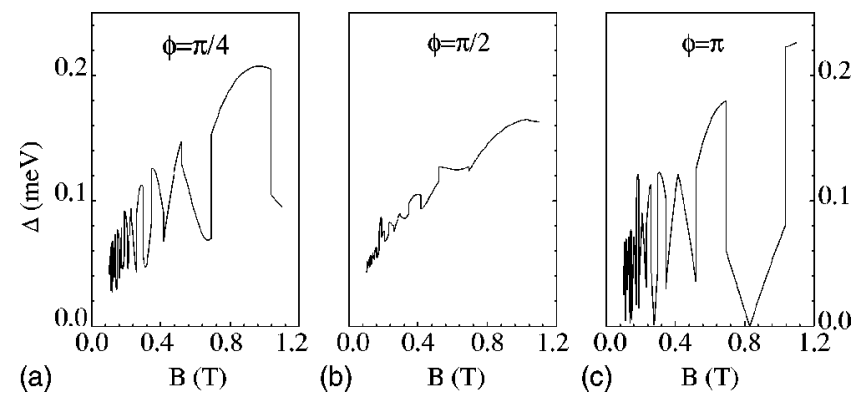

FIG. 8. Bandwidth at the Fermi energy as a function of B for different phases $\phi=\pi / 4$ (a), $\phi=\pi / 2$ (b), and $\phi=\pi$ (c) between the two modulations.
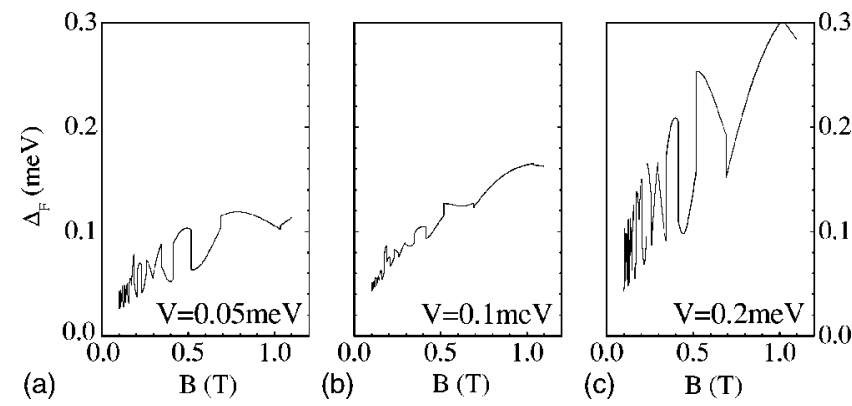

FIG. 9. The same as in Fig. 7 for different electric modulation strengths $V$, as indicated, and fixed magnetic one $\hbar \omega_{0}=0.1 \mathrm{meV}$ at phase shift of $\pi / 2$.

of the modulations. This envelope coincides with the spectrum obtained from the Harper-type equation for $\alpha=\Phi_{0} / \Phi$ integer, where $\Phi$ is the flux per unit cell. For $\alpha$ integer the analytical spectrum Eq. (16) coincides with the one obtained from the numerical solution of Eq. (15). As an approximation, one can use the spectrum for $\alpha$ integer for all magnetic fields if the small gaps of the exact spectrum are closed due to disorder. ${ }^{8}$ The flat-band condition and the bandwidth are then found from the approximate bandwidth for large $n$.

For $\alpha=q / p$ rational, the gaps in the exact spectrum, for the usual sinusoidal 2D modulations, are small and nearly close when a small level broadening is included in the calculation of the DOS whether both modulations are present or only the electric one. ${ }^{8}$ The value of $\Gamma$ needed to close the gaps depends on the modulation strength. For instance, with $V_{x}=V_{y}=0.5 \mathrm{meV}$, a width $\Gamma=1.1 \mathrm{~K}$ was necessary. ${ }^{8}$ In both cases, $\Gamma$ is quite small. Accordingly, the very interesting fine structure of the spectrum may be very difficult to observe.

As discussed in the text and illustrated in Fig. 3, the spectrum for $\alpha$ integer and a pure electric modulation is in general equivalent to that of a pure magnetic modulation if we neglect the very small terms $\propto \epsilon_{\mu}$. The same conclusion was reached in Ref. 12 for a perurbative evaluation of the spec-
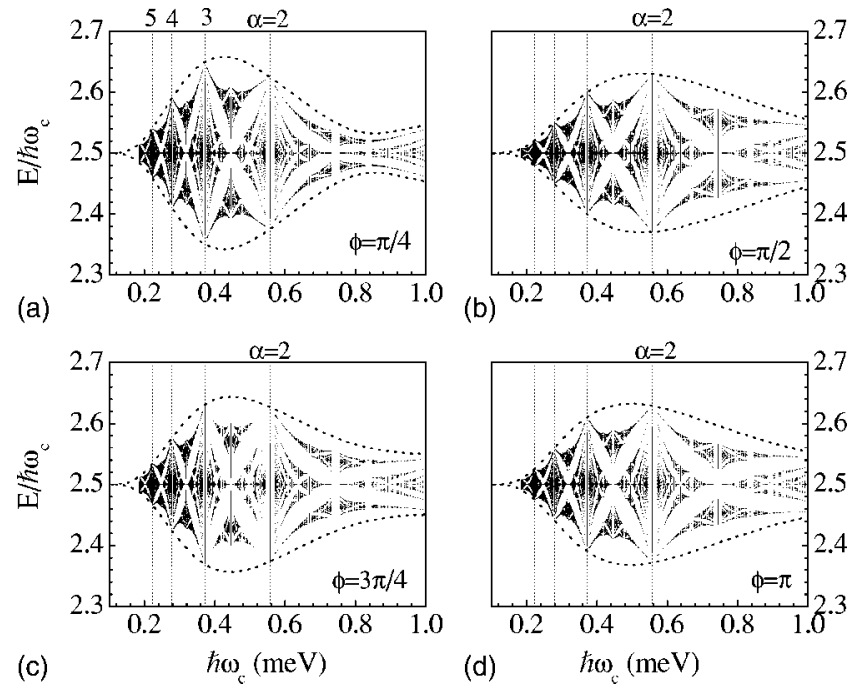

FIG. 10. Exact and approximate (dotted curves) spectrum for various phase differences between the two modulations $\phi=\pi / 4$ (a), $\phi=\pi / 2$ (b), $\phi=3 \pi / 4$ (c), and $\phi=\pi(\mathrm{d})$. 
trum. The same analogy, though less explicitly, applies to the exact spectrum obtained from Eq. (15) and shown in Fig. 5.

In line with our previous study, ${ }^{8}$ for other kinds of surface superlattices, e.g., hexagonal or trigonal, the results are similar to those presented here even when we include cross terms $\propto V_{x} V_{y} \cos \left(K_{x} x\right) \cos \left(K_{y} y\right)$ in the modulation potential.

The oscillations of the bandwidth with the magnetic field $B$, due to only an electric modulation, are in antiphase with those due to only a magnetic modulation. If both modulations are present, an additional parameter is the phase between them and the situation is more complex. The relative importance of the two modulations can be estimated by the parameter $\delta$, which is determined by the modulation strengths and the electron density. The electric modulation dominates for $\delta<1$ and the magnetic one for $\delta>1$. By changing the electron density, the Fermi energy can be shifted from the $\delta<1$ regime to the $\delta>1$ one. Alternatively, one can change the relative strengths between the electric and magnetic modulations to tune the value of $\delta$ which subsequently will influence the magnetoresistance. Similar to the case of $1 \mathrm{D}$ modulations, ${ }^{13}$ a transition between the two regimes occurs for $\delta=1$ and the commensurability oscillations of the diffusive contributions to the resistivity disappear if the two modulations are phase shifted by $\pi / 2$.

\section{ACKNOWLEDGMENTS}

This work was supported by the Canadian NSERC Grant No. OGP0121756, the Belgian Interuniversity Attraction Poles (IUAP), the Flemish Concerted Action (GOA) Programme, and the EU-CERION program.
${ }^{1}$ D. Weiss, K. von Klitzing, K. Plog, and G. Weimann, Europhys. Lett. 8, 179 (1989); R. W. Winkler, J. P. Kotthaus, and K. Ploog, Phys. Rev. Lett. 62, 1177 (1989).

${ }^{2}$ R. R. Gerhardts, D. Weiss, and K. von Klitzing, Phys. Rev. Lett. 62, 1173 (1989); C. W. J. Beenakker, ibid. 62, 2020 (1989); P. Vasilopoulos and F. M. Peeters, ibid. 63, 2120 (1989).

${ }^{3}$ D. E. Grant, A. R. Long, and J. H. Davies, Phys. Rev. B 61, 13127 (2000).

${ }^{4}$ D. Pfannkuche and R. R. Gerhardts, Phys. Rev. B 46, 12606 (1992).

${ }^{5}$ E. S. Alves, P. H. Beton, M. Henini, L. Eaves, P. C. Main, O. H. Hughes, G. A. Toombs, S. P. Beaumont, and C. D. W. Wilkinson, J. Phys.: Condens. Matter 1, 8257 (1989); H. Fang and P. J. Stiles, Phys. Rev. B 41, 10171 (1990); A. Toriumi, K. Ismail, M. Burkhardt, D. A. Antoniadis, and Henry I. Smith, ibid. 41, 12346 (1990).

${ }^{6}$ D. R. Hofstadter, Phys. Rev. B 14, 2239 (1976); F. H. Claro and G. H. Wannier, ibid. 19, 6068 (1979).

${ }^{7}$ C. Albrecht, J. H. Smet, D. Weiss, K. von Klitzing, R. Hennig, M. Suhrke, U. Rossler, V. Umansky, and H. Schweizer, Phys. Rev. Lett. 86, 147 (2001); S. Chowdhury, C. J. Emeleus, B. Milton, E. Skuras, A. R. Long, J. H. Davies, G. Pennelli, and C. R. Stanley, Phys. Rev. B 62, R4821 (2000).
${ }^{8}$ X. F. Wang, P. Vasilopoulos, and F. M. Peeters, Phys. Rev. B 69, 035331 (2004).

${ }^{9}$ S. Chowdhury, A. Long, E. Skouras, J. H. Davies, K. Lister, G. Pennelli, and C. R. Stanley, Phys. Rev. B 69, 035330 (2004).

${ }^{10}$ P. D. Ye, D. Weiss, R. R. Gerhardts, M. Seeger, K. von Klitzing, K. Eberl, and H. Nickel, Phys. Rev. Lett. 74, 3013 (1995).

${ }^{11}$ H. A. Carmona, A. K. Geim, A. Nogaret, P. C. Main, T. J. Foster, M. Henini, S. P. Beaumont, and M. G. Blamire, Phys. Rev. Lett. 74, 3009 (1995); Y. Iye, A. Endo, S. Izawa, M. Kato, and S. Katsumoto, Physica B 227, 122 (1996).

${ }^{12}$ M. C. Chang and Q. Niu, Phys. Rev. B 50, 10843 (1994); X. Wu and S. E. Ulloa, ibid. 47, 10028 (1993).

${ }^{13}$ F. M. Peeters and P. Vasilopoulos, Phys. Rev. B 47, 1466 (1993).

${ }^{14}$ F. M. Peeters, P. Vasilopoulos, and J. Shi, J. Phys.: Condens. Matter 14, 8803 (2002).

15 J. Labbé, Phys. Rev. B 35, 1373 (1987).

${ }^{16}$ R. E. Peierls, Z. Phys. 80, 763 (1933); P. G. Harper, Proc. Phys. Soc., London, Sect. A 68, 874 (1955); G. H. Wannier, Rev. Mod. Phys. 34, 645 (1962); M. Ya. Azbel', Sov. Phys. JETP 19, 634 (1964).

${ }^{17}$ N. A. Usov, Zh. Eksp. Teor. Fiz. 94, 305 (1988) [Sov. Phys. JETP 67, 2565 (1988)].

${ }^{18}$ A. H. MacDonald, Phys. Rev. B 28, 6713 (1983). 